\title{
Algorithm for Preliminary Analysis of Viscosity Behaviour of Some Aqueous Poly(ethylene oxide) Gels
}

\author{
M. TodicA*, C.V. Pop, L. Udrescu And M. Pop \\ Faculty of Physics,"Babes-Bolyai" University, M. Kogalniceanu No. 1, 400084 Cluj-Napoca, Romania \\ (Received June 7, 2010; in final form February 20, 2011)
}

\begin{abstract}
The dependence of the viscosity on temperature and shear rate was observed for some poly(ethylene oxide) gels with different polymeric concentrations. An algorithm of analysis of experimental data, based on the Arrhenius and power law models, and a mathematical function describing the dependence of the viscosity on temperature and shear rate are proposed.
\end{abstract}

PACS: $82.70 . \mathrm{Dd}, 82.70 . \mathrm{Gg}, 82.70 . \mathrm{Kj}$

\section{Introduction}

Some modern pharmaceutical products with applications in the treatment of the skin disease are based on the use of the polymeric matrix as support for the medical drugs. The advantage of such products is the possibility to apply the active substance only in the desired area of the skin, the prolonged time contact between the product and the tissue and the possibility to control the delivery rate of the active substance [1-3].

Poly(ethylene oxide) (PEO) is one of the polymers used actually for this purpose. It is characterized by a great affinity for water and the capacity to produce gel in aqueous solutions $[4,5]$. The spreading capacity and the possibility to remove the polymeric matrix from the skin after use are important properties of these products, directly correlated with the viscosity. The viscosity represents the friction force between two adjacent layers of flow and it is determined by the attractive forces acting between the particles of the fluid [6]. For the polymeric systems the viscosity is determined by the temporary or permanent junctions between the polymeric chains. These connections reduce the free motions of the macromolecules, and their stability is determined by the polymeric concentration, temperature and the rate of mechanical solicitation exerted on the sample. On the macroscopic scale these effects are reflected by the dependence of the viscosity on the temperature, concentration, and shear rate. There are many mathematical models, Binghman, Casson and power law, describing one or other of this dependence, applied to simple liquids or particularly system, but a general model, valid for a large category of samples, is very difficult to establish $[6,7]$. Usually for a given sam-

* corresponding author; e-mail: mihai.todica@phys.ubbcluj.ro ple, a particularization of the known models is requested. Islam et al. and Kim et al. described the dependence of the viscosity versus shear rate of Carbopol by power model, and the temperature dependence of the viscosity by the Arrhenius law $[8,9]$. Power model was used also by Park et al. to describe the dependence of the viscosity on the shear rate for some PEO samples [10]. Generally each of these dependences was analyzed individually and described by separate mathematical formula.

The aim of our work is to analyze the behavior of the viscosity of some aqueous dispersion of PEO and to find a single mathematical function to describe both the shear rate and temperature dependence of the viscosity. For this purpose we propose a particular algorithm of analyze of experimental data recorded at different temperatures and shear rates and we particularized two known models, the Arrhenius and power law models, to explain the dependence of the viscosity on temperature and shear rate. These dependences are expressed by a single mathematical formula.

\section{Experimental}

We used the poly(ethylene oxide) PEO 750 in our study. At low polymeric concentration, a semisolid gel is obtained by mixing the polymer with distilled water for $3-4 \mathrm{~h}$. The polymeric concentrations of the samples were $\phi=7 \%$ and $\phi=9 \%$. Homogeneous gels can be obtained only at small polymeric concentration. Above the concentration $11 \%$, a separation of phases is observed [11]. We measured the viscosity of these samples at different rotation speeds, between 0 and $200 \mathrm{rpm}$, with a Brookfield DV II Pro viscometer. The determinations are made in the temperature range $26-55^{\circ} \mathrm{C}$. The experimental error of measurements was less than $3 \%$. 


\section{Results and discussion}

For these samples the viscosity is dependent on three variables, concentration, temperature, and shear rate. Our efforts were focused to find the contribution of each variable to the viscosity. For this purpose we performed two series of measurements of viscosity, at constant temperature and then at constant shear rate. For the first series of measurements we maintained the concentration and temperature constant and we measured the viscosity as function of shear rate. For each temperature and for each polymeric concentration, the measurements were performed in the same range of shear rate.

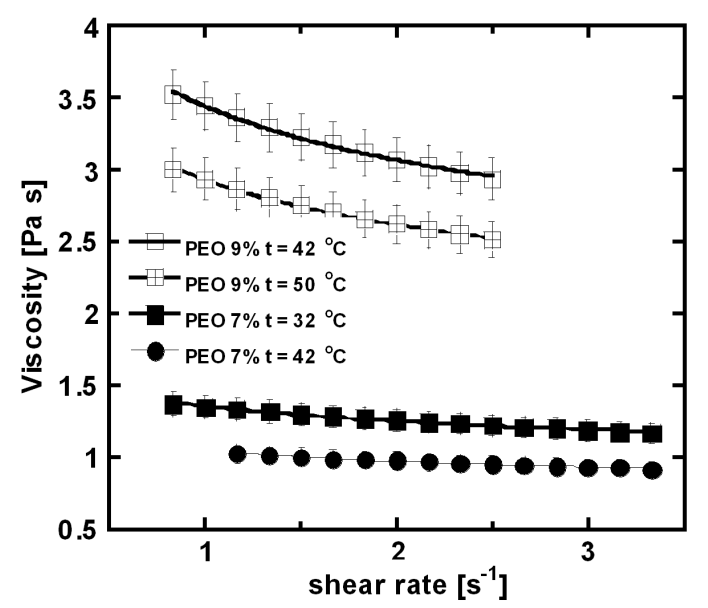

Fig. 1. Viscosity vs. shear rate curves, at different constant temperatures, for samples with polymeric concentrations $7 \%$ and $9 \%$. The parameters of the fit are: sample $\phi=7 \%, t=32{ }^{\circ} \mathrm{C}, a=1.35 \mathrm{~Pa} \mathrm{~s}^{n}, n=0.88$; $\Phi=7 \%, t=42{ }^{\circ} \mathrm{C}, a=1.04 \mathrm{~Pa} \mathrm{~s}^{n}, n=0.90$; sample $\Phi=9 \%, t=42{ }^{\circ} \mathrm{C}, a=3.43 \mathrm{~Pa} \mathrm{~s}{ }^{n}, n=0.83 ; \phi=9 \%$, $t=50^{\circ} \mathrm{C}, a=2.92 \mathrm{~Pa} \mathrm{~s}^{n}, n=0.83$.

For a given temperature and concentration we observed a decrease of the viscosity with the increase of shear rate (Fig. 1). This behavior is determined, on microscopic scale, by the alignment of polymeric chains, in the direction of flow, the decrease in number of entanglements between polymeric chains segments and modification of the shape of the microgel particles under the external mechanical solicitation [8]. Usually, one of simplest mathematical models, Casson, Binghman, power law, is used for preliminary analysis. In previous work reported on PEO samples submitted to thermal degradation, we observed similar variation of the viscosity on shear rate, and we established that the best approximation of the experimental data is obtained with the power model [11]. Similar analysis was performed with good results by Islam et al. for carbopol [8]. The equation describing this dependence is

$$
\eta=a(\dot{\gamma})^{n-1}
$$

$\eta$ represents the viscosity, $\dot{\gamma}$ represents the shear rate, $n$ is the power exponent and $a$ is a proportionality parameter.
We used this equation to approximate the dependence on shear rate of our experimental data. The data were analyzed with well known software Kaleidagraph and Origin. For a given set of data there is only a single output for the fit parameters, and the software provides the error of data approximation. Because some of our data are affected by measuring errors, we repeated the fit for all sets of data, containing the minima and maxima error values, and we watched the error of approximation offered by the computer. We considered the best fit of the experimental data the situation corresponding to the maximum rate of approximation given by the computer. In this case the curve traced by the computer passes almost through all the experimental points. The error in the calculation of parameters is less than $3 \%$ and is determined by the errors of experimental data.

For instance at $t=32^{\circ} \mathrm{C}$ and $\phi=7 \%$, the best fit of the experimental data is obtained for $n=0.88$ (Fig. 1). For other temperatures, but in the same domain of shear rate and for the same concentration, the variation of the power exponent $n$ is very small, only the parameter $a$ varies significantly. The values of these parameters are listed in Table I. For example the variation of power exponent $n$ with the temperature, for sample with concentrations $7 \%$ represents $2.2 \%$ from its minimum value, whereas the variation of parameter $a$ represents $30 \%$ from its minimum value. Similar variations can be observed also for the sample with concentration $9 \%$ (Table I). On the other hand, the small value of exponent $(n-1)$ indicates little deviation from Newtonian model. Or in Newtonian model usually the temperature dependence is contained in the proportionality factor $a$. For the sake of simplicity the interest is to find simple mathematical functions, and if possible independent function, to express the dependences on different variables (temperature and shear rate).

TABLE I

Values of parameters $a$ and $n$ of power model.

\begin{tabular}{c|c|c|c|c}
\hline \hline$\phi$ & $t\left[{ }^{\circ} \mathrm{C}\right]$ & $a\left[\mathrm{~Pa} \mathrm{~s}^{n}\right]$ & $n$ & $\bar{n}$ \\
\hline \multirow{3}{*}{$7 \%$} & 32 & 1.35 & 0.88 & \\
& 37 & 1.17 & 0.89 & 0.89 \\
& 42 & 1.04 & 0.90 & \\
\hline \multirow{3}{*}{$9 \%$} & 42 & 3.43 & 0.83 & \\
& 50 & 2.92 & 0.83 & 0.84 \\
& 55 & 2.61 & 0.86 &
\end{tabular}

Taking into account the small variation of power exponent $n$ (included in the error limit of experimental data), we can consider this parameter constant for a given concentration. For further mathematical analysis, at constant concentration, we used the average value of $n$ calculated for many temperatures. The analyze of experimental data corresponding to the sample with $9 \%$ concentration, reveals similar behavior, but the value of $n$ is different. The first conclusion resulting from this analysis 
is the independence of parameter $n$ on the temperature (in the domain of temperatures of our study), at constant concentration. Its variation is determined mainly by the variation of concentration. So we can consider $n$ a function of concentration, $n(\phi)$.

The viscosity of highly interconnected systems as the macromolecular solutions or gels is a very complex mechanism that requests the adaptation of known models for each category of molecular system and for each domain of temperature, shear rate and concentrations. Power model is related to the interconnected systems and the power exponent $n$ characterises this interconnectivity. If this parameter depends only on concentration and not on the temperature, this fact clearly demonstrates that the variation of the viscosity with the shear rate is determined mainly by the modification of the connections between the polymeric chains. Modification of the concentration leads to a modification of degree of interconnectivity between the chains. From mathematical point of view $n$ becomes a function of concentration. However, in our experiments we explored a small domain of concentration that leads difficult the task to establish the dependence law of power exponent $n$ with the concentration. We can only calculate its values corresponding to our concentrations. The best values are $n=0.89$ for $7 \%$ concentration and $n=0.84$ for $9 \%$ concentration and the best fit for some temperatures with Eq. (1) is shown in Fig. 1.

For a given concentration and temperature Eq. (1) provides values of the parameter $a$ independent of shear rate. For other temperatures, but for the same concentration, the values of parameter $a$ obtained with Eq. (1), are different (Table I).

At constant concentration but at different temperatures, the conformations of the polymeric chains change due to the thermal agitation. Every change in conformation requests a quantity of energy, the activation energy, like in the model of the potential barrier energy (often used for liquids with simplest molecules). In the known models, this effect is reflected by the dependence on temperature of the proportionality factor $a$.

Taking into account our results, we can conclude that the parameter $a$ depends on temperature $a=a(T)$. If we change the concentration, but we keep the same temperature and the same domain of shear rate, we find different values for this parameter, i.e. $a=1.04 \mathrm{~Pa} \mathrm{~s}^{n}$ for concentration $7 \%$ at $42{ }^{\circ} \mathrm{C}$ and $a=3.43 \mathrm{~Pa} \mathrm{~s}^{n}$ for concentration $9 \%$ at $42{ }^{\circ} \mathrm{C}$ (Table I). From these observations we can conclude that the parameter $a$ is a function of temperature and concentration. We can write $a=a(T, \phi)$.

Our interest is to separate the contribution of these variables, temperature and concentration, in the mathematical expression of the function $a(T, \phi)$. For this aim we measured the viscosity at constant shear rate and constant concentration at different temperatures. For a given concentration and rotation speed, i.e. $7 \%$, and $50 \mathrm{rpm}$, the viscosity decreases with the temperature, Fig. 2. We observed the same behavior for higher rota- tion speeds, but the values of the viscosity are smaller. At constant rotation speed the factor $(\dot{\gamma})^{n-1}$ is constant in Eq. (1), $(\dot{\gamma})^{n-1}=B=$ const, and the variation of the viscosity is expressed only by the function $a(T, \phi)$ :

$$
\eta=a(T, \phi) B \text {. }
$$

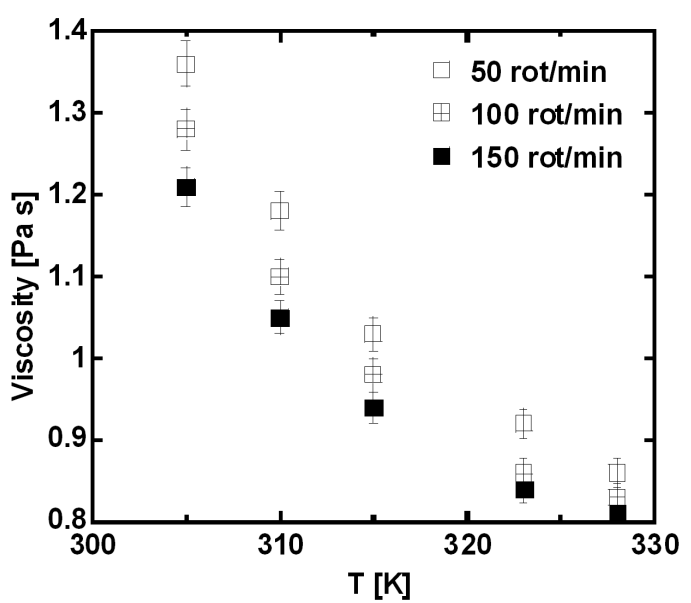

Fig. 2. Viscosity vs. temperature curves, at three constant shear rates, for sample with polymeric concentration $7 \%$.

Usually the dependence of the viscosity on the temperature is expressed by the Arrhenius law [12]:

$$
\eta=C(\phi) \exp \left(\frac{E_{\mathrm{a}}}{R T}\right) B
$$

$E_{\mathrm{a}}$ is the activation energy of the flow, $R$ is the universal constant of the gases and $C(\phi)$ is a proportionality factor containing the contribution of concentration. By identification, from Eq. (2) and Eq. (3) we can write

$$
a(T, \phi)=C(\phi) \exp \left(\frac{E_{\mathrm{a}}}{R T}\right) .
$$

In this equation the contributions of temperature and concentration to the function $a(T, \phi)$ are separated. The $E_{\text {a }}$ is not known but we can calculate its value from the Arrhenius plot of viscosity versus temperature, at constant concentration.

A typical representation for the sample with concentration $9 \%$ is shown in Fig. 3. We calculated the values of $E_{\mathrm{a}}$ for three different rotation speeds, 50, 100, $150 \mathrm{rpm}$, in order to observe eventual dependence of $E_{\mathrm{a}}$ on the shear rate. For a given concentration the values of $E_{\mathrm{a}}$ calculated at these rotation speeds are very close to each other indicating no dependence of this parameter on the shear rate. For this reason we used the average value of $E_{\mathrm{a}}$ calculated at these rotation speeds, $\bar{E}_{\mathrm{a}}=15.4 \mathrm{~kJ} / \mathrm{mol}$ for the concentration $7 \%$. Similar behavior was observed for the concentration $9 \%$, but the value of $E_{\mathrm{a}}$ is different, $\bar{E}_{\mathrm{a}}=17.0 \mathrm{~kJ} / \mathrm{mol}$. These values are listed in Table II. Now we can describe the variation of $a(T, \phi)$ with the temperature by introducing the average 


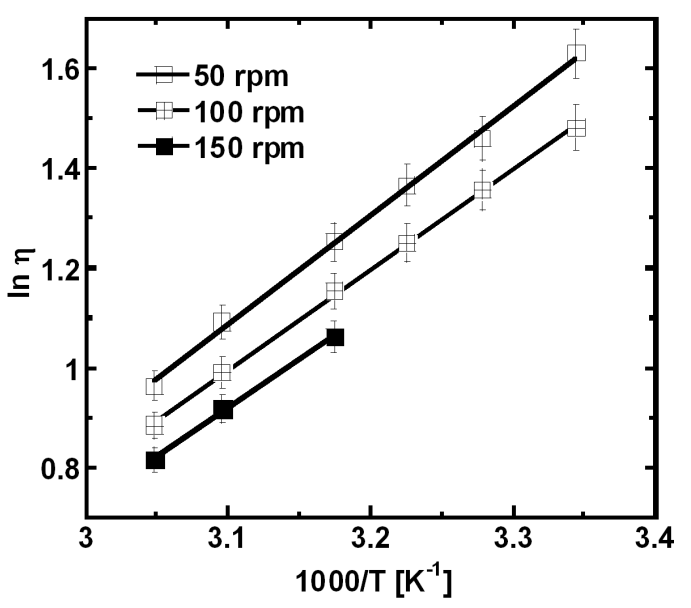

Fig. 3. Arrhenius plot of viscosity data, at three constant shear rates, for sample with polymeric concentration $9 \%$. The values of activation energy resulting from this plot are: $E_{\mathrm{a}}=18.0 \mathrm{~kJ} / \mathrm{mol}$ at $50 \mathrm{rpm}$, $E_{\mathrm{a}}=16.8 \mathrm{~kJ} / \mathrm{mol}$ at $100 \mathrm{rpm}, E_{\mathrm{a}}=16.2 \mathrm{~kJ} / \mathrm{mol}$ at $150 \mathrm{rpm}$.

values of $E_{\mathrm{a}}$ in Eq. (4). Combining the two dependences, on temperature, Eq. (4), and shear rate, Eq. (1), we can write a new equation for the viscosity

$$
\eta(\phi, T, \dot{\gamma})=C(\phi) \exp \left(\frac{E_{\mathrm{a}}(\phi)}{R T}\right)(\dot{\gamma})^{n-1} .
$$

This equation describes in a single mathematical formula the dependence of viscosity on temperature, concentration and shear rate. The contribution of shear rate is contained in the power factor. The contributions of concentration is contained in the functions $C(\phi), n$ and $E_{\mathrm{a}}$ and the contribution of temperature in the exponential factor. Although this equation was obtained from separate analysis of the dependence of viscosity on temperature and shear rate, its utility results from the possibility to approximate the experimental data whenever the temperature, concentration or shear rate, are variable.

TABLE II

Values of parameters $C(\phi)$ and $E_{\mathrm{a}}$ in Eqs. (3) and (5).

\begin{tabular}{c|c|c|c|c|c|c}
\hline \hline$\phi$ & $\begin{array}{c}t \\
{\left[{ }^{\circ} \mathrm{C}\right]}\end{array}$ & $\begin{array}{c}C(\phi) \\
{\left[\mathrm{Pa} \mathrm{s}^{n}\right]}\end{array}$ & $\begin{array}{c}a \\
{\left[\mathrm{~Pa} \mathrm{~s}^{n}\right]}\end{array}$ & $\begin{array}{c}\bar{C} \\
{\left[\mathrm{~Pa} \mathrm{~s}^{n}\right]}\end{array}$ & $\begin{array}{c}\bar{E}_{\mathrm{a}} \\
{[\mathrm{kJ} / \mathrm{mol}]}\end{array}$ & $\bar{n}$ \\
\hline \multirow{3}{*}{$7 \%$} & 32 & 0.0030 & 1.26 & & & \\
& 37 & 0.0029 & 1.14 & 0.0029 & 15.4 & 0.89 \\
& 42 & 0.0028 & 1.04 & & & \\
\hline \multirow{4}{*}{$9 \%$} & 42 & 0.0051 & 3.37 & & & \\
& 50 & 0.0051 & 2.87 & 0.0051 & 17.0 & 0.84 \\
& 55 & 0.0051 & 2.60 & & &
\end{tabular}

We have shown already that $n$ and $E_{\mathrm{a}}$ are constant for a given concentration. When we analyze the dependence of the viscosity on shear rate, for a given temperature, at constant concentration, the function $C(\phi)$ must be constant. To verify this supposition, we used the average values of $E_{\mathrm{a}}$ and $n$ calculated at constant concentration, and we analyzed the variation of experimental data $\eta(T, \dot{\gamma})$ as function of shear rate, with Eq. (5). The values of parameter $C(\phi)$ resulting from this fit are listed in Table II for both concentrations. We found that $C(\phi)$ is practically constant for all the temperatures and shear rates, at constant concentration. Further we considered only its average value (Table II). Its value changes with the concentration. This result confirms again the supposition that $C(\phi)$ is a function of concentration. The fit of experimental data with Eq. (5) for concentration $9 \%$ is shown in Fig. 4, and for concentration $7 \%$ in Fig. 5.

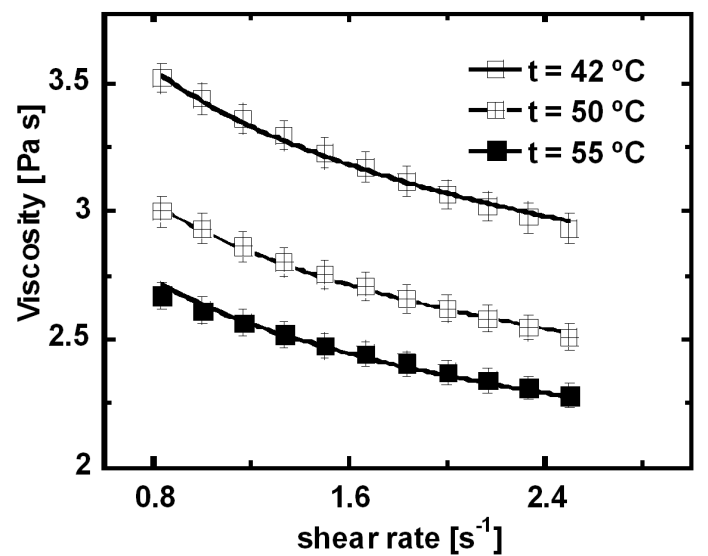

Fig. 4. Fit with Eq. (5) of experimental data representing the variation of the viscosity vs. shear rate, at different constant temperatures, for the sample with polymeric concentration $9 \%$. The parameters of the fit are: at $t=42^{\circ} \mathrm{C}, C(\phi)=0.0051 \mathrm{~Pa} \mathrm{~s}^{n}, E_{\mathrm{a}}=17.0 \mathrm{~kJ} / \mathrm{mol}$, $n=0.84 ;$ at $t=50^{\circ} \mathrm{C}, C(\phi)=0.0051 \mathrm{~Pa} \mathrm{~s}{ }^{n}$, $E_{\mathrm{a}}=17.0 \mathrm{~kJ} / \mathrm{mol}, n=0.84$; at $t=55^{\circ} \mathrm{C}, C(\phi)=$ $0.0051 \mathrm{~Pa} \mathrm{~s}^{n}, E_{\mathrm{a}}=17.0 \mathrm{~kJ} / \mathrm{mol}, n=0.84$.

We calculated also the values of parameter $a(T, \phi)$ with Eq. (4) and the average values of $E_{\mathrm{a}}$ and $C(\phi)$ (Table II). The maximum error in the calculation of this parameter is $5 \%$, determined by the use of average values of parameters $E_{\mathrm{a}}$ and $n$ to calculate $C(\phi)$ with Eq. (5). The values of parameter $a(T, \phi)$ calculated by this way are very close (in the limit of the error analysis) to the values resulting from the direct fit of data with Eq. (1) and reported in Table I.

In Eq. (1) the parameter a represents a free parameter without any constraint imposed by a given mathematical function (depending on different physical variables, concentration, temperature, shear rate). It can have any values, in order to approximate as well as possible the experimental data, but in this case, its physical meaning is not relevant. The situation is different in Eq. (4), where this parameter is correlated with the temperature and concentration. Although its values are little different from those calculated directly (Table I), its physical meaning is more realistic because its dependence on tem- 


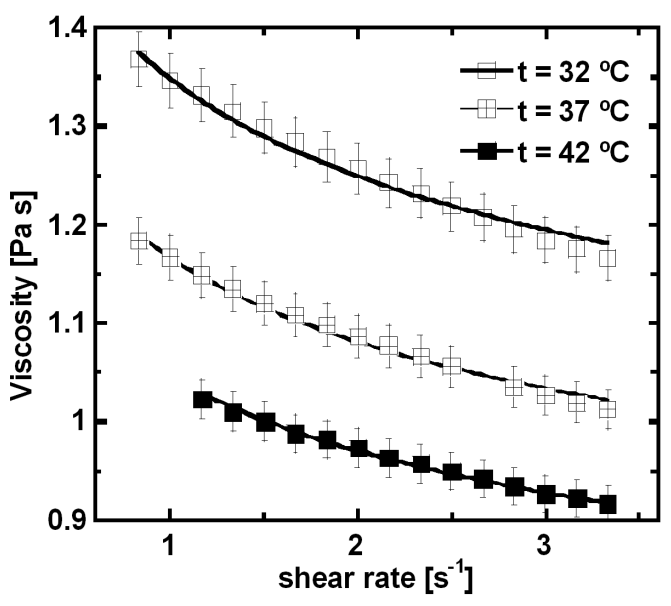

Fig. 5. Fit with Eq. (5) of experimental data representing the variation of the viscosity vs. shear rate, at different constant temperatures, for the sample with polymeric concentration $7 \%$. The parameters of the fit are: at $t=32^{\circ} \mathrm{C}, C(\phi)=0.0030 \mathrm{~Pa} \mathrm{~s}{ }^{n}, E_{\mathrm{a}}=15.4 \mathrm{~kJ} / \mathrm{mol}$, $n=0.89 ;$ at $t=37^{\circ} \mathrm{C}, C(\phi)=0.0029 \mathrm{~Pa} \mathrm{~s}^{n}$, $E_{\mathrm{a}}=15.4 \mathrm{~kJ} / \mathrm{mol}, n=0.89$; at $t=42{ }^{\circ} \mathrm{C}, C(\phi)=$ $0.0028 \mathrm{~Pa} \mathrm{~s}^{n}, E_{\mathrm{a}}=15.4 \mathrm{~kJ} / \mathrm{mol}, n=0.89$.

perature and concentration is expressed by a mathematical function constructed on the basis of known physical models. The main advantage of Eq. (5) is the possibility to separate the contribution of different variables (shear rate, concentration, temperature) to the viscosity. A separation of contribution of each variable allows a better explanation of the mechanism governing the viscosity. A function depending only on concentration can be easier correlated with the interconnected character of the system (the existence of the temporary or permanent junctions between the particles of the system); a function depending on temperature is easy correlated with the barrier energy potential model. In this way our equation can be helpful to the study of the polymeric flow. It represents a preliminary approximation of the polymeric viscosity applied to a limited domain of temperature, concentration and shear rate, for category of samples close to our system. Wide use of this equation imposes the analysis of a large category of systems and a wide domain of variations of physical parameters, temperature, shear rate, concentration.

\section{Conclusions}

Measurements performed on aqueous PEO samples show a dependence of the viscosity on three variables, temperature, concentration, and shear rate. This dependence is analyzed on the basis of the Arrhenius and power model. A particular algorithm of mathematical analysis of experimental data allows a separation of the contribution of each variable to the viscosity. Such separation allow a better correlation between the mechanisms governing the flow (local modification of the conformation of polymeric chain or modification of the interconnections between the polymeric chains) and the variation of viscosity. These contributions are included into a single mathematical formula. The experimental data can be well approximated with this formula.

\section{References}

[1] N. Lambov, N. Dimitrov, S. Tsankov, Pharmazie 52, 790 (1997).

[2] H. Savaş, O. Güven, Int. J. Pharm. 224, 151 (2001).

[3] L. Maggi, L. Segale, M.L. Torre, M.E. Ochoa, U. Conte, Biomaterials 23, 1113 (2002).

[4] H. Kojima, K. Yoshihara, T. Sawada, H. Kondo, K. Sako, Europ. J. Pharmaceut. Biopharmaceut. 70, 556 (2008).

[5] E. Dinte, R.I. Iovanov, S.E. Leucuta, D. Ionescu, Farmacia LIV, 29 (2006).

[6] G. Astaria, G. Marrucci, Principles of Non-Newtonian Fluid Mechanics, McGraw-Hill, New York 1974.

[7] S. Tamburic, D.Q.M. Craig, J. Control. Rel. 32, 59 (1995).

[8] M.T. Islam, N. Rodríguez-Hornedo, S. Ciotti, C. Ackermann, Pharmaceut. Res. 21, 1192 (1995).

[9] J.Y. Kim, J.Y. Song, E.J. Lee, S. Park, Colloid Polymer Sci. 281, 614 (2003).

[10] S.-W. Park, B.-S. Choi, J.-W. Lee, Korea-Australia Rheol. J. 17, 199 (2005).

[11] M. Todica, C.V. Pop, Chin. Phys. Lett. 26, 078201 (2009).

[12] J. Ferguson, Z. Kemblowski, Applied Fluid Rheology, Elsevier, London 1992. 